\title{
AURICULOTERAPIA: MÉTODO ALTERNATIVO PARA O COMBATE DO ESTRESSE
}

\author{
AURICULOTHERAPY: ALTERNATIVE METHOD FOR STRESS \\ FIGHTING
}

\author{
Viganó, J.R ${ }^{1}$, Cerutti, M.L ${ }^{2}$, Dullius, $\mathrm{C}^{3}, \mathrm{Bado}^{3}, \mathrm{~L}$, Valente, $\mathrm{C}^{4}$. \\ Afiliações: 1- Departamento de Farmácia da Universidade Regional de Blumenau- FURB. 2- Faculdade de Medicina da Universidade \\ Regional de Blumenau- FURB. 3 - Centro de Ciências da Saúde da Universidade Estadual do Oeste do Paraná- UNIOESTE. 4- \\ Departamento de Ciências Naturais da Universidade Regional de Blumenau- FURB. \\ Endereço do autor de correspondência: Rua Antônio da Veiga, 140- Itoupava Seca, Blumenau, Santa Catarina, Brasil. CEP: 89030-903. \\ carolvalente11@gmail.com.
}

\section{Resumo}

O presente estudo procura demonstrar a auriculoterapia como um método alternativo e complementar para a redução dos níveis de estresse, considerando que esse é um fator de risco para o desenvolvimento de patologias. Teve como objetivo realizar uma revisão bibliográfica por meio de materiais publicados nas bases de dados Scielo, PubMed e Google Acadêmico, além de livros sobre o tema para a fundamentação teórica. Foram selecionados e sintetizados em um quadro com 10 trabalhos que realizaram a prática de auriculoterapia para a redução dos níveis de estresse. Constatou-se que a maioria dos trabalhos compararam tratamentos com agulhas semipermanentes e sementes, seguido de trabalhos que compararam a aplicação da técnica por meio de tratamento individualizado e tratamento com pontos protocolares. Observou-se que a maioria dos estudos utilizaram como pontos principais os pontos Shenmen, Rim e Tronco Cerebral, além do Yang do Fígado 1 e 2. A população de estudo desses trabalhos foi quase que unanime para profissionais da área de saúde, com predomínio do sexo feminino. Apesar de cada estudo apresentar um objetivo específico, fica clara a eficácia da auriculoterapia como método alternativo para o combate do estresse.

Palavras-chave: Estresse Ocupacional; Auriculoterapia; Terapias Complementares.

\section{Abstract}

The current study aims to demonstrate auriculotherapy as an alternative and complementary method for the reduction of stress levels, considering this is a risk factor for the development of pathologies. The purpose was to perform a bibliographical review through materials published in the Scielo, PubMed and Google Academic databases, as well as books on the subject for the theoretical foundation. A total of 10 studies were selected and synthesized in a table that performed auriculotherapy for the reduction of stress levels. It was found that most of the studies compared treatments with semipermanent needles and seeds, followed by studies comparing the application of the technique by means of individualized treatment and treatment with protocol points. It was observed that most of the studies used as points Shenmen, Rim and Brainstem, besides Yang of Liver 1 and 2. The study population of these works was almost unanimous for health professionals, with predominance female. Although each study has a specific objective, it is clear the effectiveness of auriculotherapy as an alternative method to combat stress.

Keywords: Occupacional Stress; Auriculotherapy; Complementary Therapies. 


\section{Introdução}

O termo estresse foi utilizado pela primeira vez na literatura em aproximadamente 1936, por Selye para descrever a adaptação do corpo humano às mais diversas situações do diaa-dia, motivo que pode levar à queda da homeostase, desequilibrando o organismo e gerando doenças ${ }^{1}$

Alguns dos fatores que levam a esse desequilíbrio podem ser citados como sobrecarga no trabalho, problemas familiares, falta de motivação, patologias em geral, depressão, sentimentos de infelicidade, falta de reconhecimento pessoal, violência urbana, salários baixos, entre outros. ${ }^{2}$

O estresse se desenvolve em três fases: a fase de alerta onde o indivíduo é exposto ao agente estressor, fazendo com que o organismo se prepare para reagir a esse agente, a segunda fase conhecida como fase de resistência, fase na qual o agente continua a interferir no indivíduo por um longo período de tempo e faz com que o organismo utilize suas energias para combatê-lo, e pôr fim a terceira fase, a fase de exaustão, onde o estresse já está instalado de forma duradoura, esgotando a reserva energética do organismo. ${ }^{3}$
Um exemplo clássico de estresse no diaa-dia é a conhecida síndrome de burnout ou síndrome do esgotamento profissional, descrito pelo desgaste tanto físico quanto emocional, motivado pela diminuição de energias e pelo desinteresse em enfrentar os problemas provenientes do trabalho. ${ }^{4}$

Em busca da diminuição do estresse, sensação de bem-estar e uma melhor qualidade de vida, estão as estratégias conhecidas como coping, e dentre essas inclui-se a técnica de auriculoterapia exercida com base na Medicina Tradicional Chinesa. ${ }^{5} 6$

A Medicina Tradicional Chinesa teve sua origem na China, e toma como base o indivíduo como um ser integral, analisando-o como um todo. Esse sistema utiliza de fundamentos como a teoria yin-yang, que descreve duas energias opostas, porém complementares, a teoria dos cinco movimentos onde confere a todos os fenômenos, bem como ao corpo humano as cinco energias (madeira, fogo, terra, metal e água), e a teoria dos órgãos e vísceras Zang Fu que correspondem a campos energéticos do organismo humano. ${ }^{7}$

Dessa maneira, a auriculoterapia visa o reequilíbrio energético em patologias através da inserção de agulhas, esferas de prata, ouro, 
cristal ou mostarda em pontos estratégicos do pavilhão auricular, sendo feita a escolha dos pontos e consequentemente o diagnóstico através da anamnese e análise desse microssistema. $^{8}$

Tendo em vista que o estresse já é considerado um problema global devido ao estilo de vida da população e por esse ser um dos fatores que ocasionam graves patologias, acredita-se que deva ser estimulada cada vez mais a utilização de meios para o combate desse mal, portanto o presente trabalho teve como como objetivo buscar por meio de revisão bibliográfica estudos que aplicaram a técnica de auriculoterapia, extraindo as principais informações na utilização desse método como prática alternativa de tratamento e controle dos níveis de estresse.

\section{Métodos}

Trata-se de um estudo executado a partir de um levantamento bibliográfico integrativo, buscando agrupar e sintetizar as informações e resultados das experiências práticas vivenciadas por outros autores.

Partindo do objetivo deste trabalho, foram utilizados os seguintes bancos de dados, para encontrar os trabalhos sobre a temática em questão: google acadêmico, Scientific Electronic Library Online (SciELO) e US National Library of Medicine (PubMed), empregando como palavras de busca os termos "estresse", "auriculoterapia" "Medicina Tradicional Chinesa", além de livros sobre a temática.

Como resultado final da pesquisa, foram selecionados um total de 10 trabalhos publicados, incluindo artigos, teses e/ou dissertações, em inglês e português, que aplicaram a técnica de auriculoterapia para a redução dos níveis de estresse e que possibilitaram definir a resposta do objetivo.

Com a definição dos estudos a serem avaliados, pode-se retirar informações e sintetizá-las em um quadro, para assim interpretar os resultados e discutir aspectos pertinentes ao objetivo deste trabalho.

\section{Desenvolvimento}

\section{ESTRESSE}

O estresse é uma palavra com origem do latim, descrita pela primeira vez na literatura em termos da física e engenharia, para nomear as forças atuantes sobre alguma resistência, e representar a carga suportável por um 
determinado material antes de sua ruptura. Contudo, essa palavra vem sendo usada desde o século XVII para descrever um estado de adversidade ou aflição, porém somente em 1936 o Dr. Hans Selye usou esse termo para referir-se a estados de tensão patogênico do organismo. A partir do século $\mathrm{XX}$ foram registradas investigações sobre os efeitos desse estado, e considerado então como um mal dos tempos que acomete a saúde física e mental das pessoas. ${ }^{9} 10$

O termo estresse é designado à adaptação do organismo a uma situação que possa ser ameaçadora a sua vida e ao seu equilíbrio, sendo considerado um aspecto normal e necessário para ocasiões de risco, pois faz com que o indivíduo se torne atento as mais diversas situações em que se apresente. ${ }^{11}$ Porém, uma sobrecarga nesta adequação pode se tornar prejudicial, pois poderá ocorrer uma quebra da homeostase do corpo, processo que o organismo sofre para adquirir um equilíbrio constante, provocada pelas reações biológicas que acometem a fisiologia biológica, física e psicológica do organismo. ${ }^{12} 13$

Os agentes estressores são diversos fatores que levam a uma extrema pressão capaz de desenvolver o estresse, e irá depender da sua intensidade variando de acordo com sua origem que pode ser emocional, ambiental, biológica e física. ${ }^{14}$

Problemas relacionados ao trabalho e família são os principais agentes estressores descritos, podendo-se citar o trabalho executado de forma excessiva, dificuldades de relacionamento entre a equipe de trabalho, falta de reconhecimento profissional, remuneração baixa, a auto cobrança, entre outros. Os efeitos da exposição frequente a esses agentes são observados pelos sentimentos de ansiedade, irritação, impaciência, conflitos internos além de baixo rendimento profissional. ${ }^{2}$

Outras causas frequentes para a instalação do estresse são as situações de perigo real, geralmente relacionado com a vida das grandes cidades, como assaltos, trânsito, desemprego, violência, entre outros (estressor externo) ou por pensamento negativos e ameaças imaginárias (estressor interno). ${ }^{15}{ }^{\prime} 16$

O estresse é um fenômeno que ocorre em momentos de extrema irritação, medo, excitação, confusão ou até mesmo euforia, podendo se desenvolver em problemas de saúde como doenças cardiovasculares, câncer, infecções, acarretando em danos físicos. Podendo afetar o sistema cognitivo causando insatisfação em atividades realizadas no dia-a- 
dia e problemas de concentração, causar sintomas emocionais como ansiedade, depressão e raiva, além de gerar sintomas somáticos (dores de cabeça, sudorese e tonturas) e sintomas comportamentais observados no uso abusivo de drogas, diminuição de produtividade, e a ausência frequente no ambiente de trabalho. ${ }^{17}$

Um dos principais problemas do estresse é a hiperfunção da glâdula supra-renal decorrente dos estímulos do agente estressor, levando a um aumento na liberação de hormônios como o cortisol, podendo ocasionar situações de descontrole pessoal. ${ }^{18}$

Para Selye ${ }^{3}$ o estresse apresenta-se em três fases: a fase de alerta (fase inicial) onde a pessoa se depara com o agente estressor e o organismo se prepara para agir contra esse agente, onde as funções orgânicas sofrem alterações, devido a ativação do hipotálamo, pelo estado de ansiedade e tensão, a hipófise então capta a mensagem do hipotálamo e envia ao sangue mensagens químicas para que o organismo reaja ao perigo. A partir daí a hipófise emite mensagens instantâneas à glândula suprarrenal para secreção de adrenalina e cortisol. Dessa forma o aumento de hormônio nas glândulas endócrinas, aceleração cardíaca e respiratória, sentidos aguçados, esfriamento de mãos e pés são sintomas da fase de alerta, que podem desaparecer em curto prazo com a eliminação do agente estressor, restaurando o equilíbrio do organismo. Caso isso não ocorra e os sintomas persistam, o estresse atinge a segunda fase, a fase de resistência. ${ }^{3}$

$\mathrm{Na}$ segunda fase, o estressor interage com o indivíduo por um período prolongado, fazendo com que o corpo tente se adaptar àquela situação, havendo um gasto de energia em excesso para um reequilíbrio, apresentando sintomas de desgaste físico, problemas de memória, perda de cabelo, aftas, irritação, redução de libido, alergias, baixa imunidade entre outros. Quando as tentativas de conseguir um reequilíbrio falharem, e a energia adaptativa se esgotar o indivíduo entra na fase de exaustão, o estresse se torna intenso, e apresenta sintomas de hipertensão, infarto, gastrite crônica e úlceras. ${ }^{3}$

Lipp $^{19}$ escreve um novo modelo para o estresse, incluindo uma quarta fase, a chamada fase de quase exaustão, em que se enquadram aspectos de enfraquecimento do indivíduo devido ao desgaste em tentar se adaptar as causas do estresse, porém a pessoa ainda consegue socializar, e realizar suas atividades do dia-a-dia por certo tempo. 
Para Lazarus, Folkman ${ }^{20}$ as formas de enfrentamento do indivíduo diante de um estressor também influenciam na atuação do estresse, e não apenas a exposição perante o agente estressor. Dito isso, pode-se classificar o estresse de forma positiva (eustresse), ou seja, um tipo de estresse que não leva a pessoa a um estado patológico, é a resposta conveniente ao estressor, traz sentimentos prazerosos e aumentam a capacidade de realizar tarefas, já no estresse negativo (distresse) ocorre reações fisiológicas causadas pela tensão acarretando em doenças físicas e psicológicas..$^{21^{\prime}} 22^{\prime} 23$

Para exemplificar esse processo de estresse, Duarte et al. ${ }^{24}$ citam a síndrome de burnout ou a síndrome do esgotamento profissional subsequente à exposição prolongada ao agente estressor, apresentando características como esgotamento físico e emocional quando o trabalhador não encontra mais meios para encarar os conflitos provenientes de sua profissão. ${ }^{4}$

Meneghini et al. $^{25}$ relata os aspectos organizacionais, de administração, do sistema de trabalho e da qualidade das relações humanas como os principais motivos desencadeadores do estresse no ambiente de trabalho. Robbins ${ }^{26}$ descreve alguns exemplos desses aspectos como sendo as tensões a fim de se evitar erros, cumprimento de tarefas em determinado período de tempo, sobrecarga de trabalho, colegas desagradáveis, chefes exigentes, empatia com colegas, entre outras.

Resultados de uma pesquisa feita pela Associação Internacional do Estresse no Brasil (ISMA-BR) em 2012, mostraram que 70\% da população brasileira que está no mercado de trabalho sofrem com problemas de estresse. ${ }^{27}$ Kurebayashi et al. ${ }^{13}$ aponta uma porcentagem de aproximadamente $90 \%$ de presença do estresse na população mundial.

Para Lazarus, Folkman ${ }^{20}$ o estresse é a resposta física e mental ocasionada por estímulos externos que permite a superação e a adequação de tal situação pelo indivíduo, sendo que o meio que o indivíduo busca para enfrentar esses estímulos é chamado de coping.

O coping para Guido et al. ${ }^{5}$ é descrito como os recursos encontrados tanto por práticas de saúde, religião, hobbies e recursos financeiros e materiais, ou seja, uma ação intencional após a percepção do agente estressor, para manter a saúde mental, física e social em equilíbrio.

Seguindo esse contexto, há um crescente interesse no uso das práticas alternativas e complementares como estratégia de coping. 
Dentre as técnicas enquadradas nessas práticas estão as técnicas de relaxamento, meditação, exercícios de respiração, alimentação balanceada e saudável, lazer, exercícios físicos, sono apropriado, técnicas da Medicina Tradicional Chinesa (MTC), como por exemplo, a auriculoterapia..$^{28} 29$

As práticas alternativas e complementares visam ativar por meios naturais a prevenção da saúde, de forma segura e eficaz. Incentivando essas técnicas a Organização Mundial da Saúde (OMS) implementou o Programa de Medicina Tradicional no final da década de 70. Porém, no Brasil essa abordagem só se tornou legítima e institucional na década de 80 especialmente após a criação do Sistema Único de Saúde (SUS), que fornece acesso a esse tipo de tratamento na rede pública de saúde através da Política Nacional de Práticas Integrativas e Complementares no SUS (PNPIC) pelo Ministério da Saúde. ${ }^{30}$

Dados do Ministério da Saúde ${ }^{30}$ trazem como data marcante para a implementação das práticas alternativas e complementares a $8^{\mathrm{a}}$ Conferência Nacional de Saúde (CNS) em 1986, sendo que dois anos após (1988) a Comissão Interministerial de Planejamento (CIPLAN) firmou as orientações para o atendimento com essas práticas. $^{7}$ Porém, apenas em 2003, foi determinada a instalação das PNPIC no SUS, através do relatório final da $12^{\mathrm{a}} \mathrm{CNS}$. Sendo que a Portaria 971 referente à PNPIC foi aprovada somente no dia 03 de maio de $2006 .^{30}$

Luz $^{31}$ menciona que a intensificação na procura de novas estratégias de cuidado com a saúde se deve principalmente às desigualdades sociais, onde as carências financeiras e as elevadas horas de trabalho ecoam na saúde física e mental das pessoas, levando a uma série de doenças. Segundo Luz ${ }^{31}$ uma segunda causa para o aumento dessas novas estratégias, a chamada “crise da medicina" na qual ele salienta sobre a difícil relação entre médico e paciente, a comercialização de tratamentos, entre outros. Já Souza, Luz ${ }^{32}$ trazem um terceiro motivo: a mudança cultural, ou seja, a realização de práticas do oriente no ocidente.

A PNPIC proporciona aos pacientes uma oportunidade de melhoria de vida e promoção de saúde por meio de terapias como homeopatia, plantas medicinais e fitoterapia, MTC e acupuntura que estimula pontos anatômicos por meio de agulhas para a promoção de saúde, medicina antroposófica e termalismo social/crenoterapia. ${ }^{33^{\prime} 7}$ 
Dados do Sistema de Informações Ambulatoriais (SIA/SUS) mostraram que no ano de 2003 houve 181.983 consultas na área de acupuntura, com maior prevalência na região Sudeste do país, estando presente em 19 estados, e localizada em 107 municípios brasileiros. ${ }^{7}$

\section{MEDICINA TRADICIONAL CHINESA}

Apesar de a Medicina Tradicional ter sido introduzida pela OMS apenas no final da década de 70 , existem relatos séculos antes de Cristo, sobre histórias de curas através de técnicas de punção para restabelecer um equilíbrio, apesar de todas as investigações científicas e estudo anatômicos precários na época. ${ }^{34}$

Kurebayashi ${ }^{35}$ descreve que apesar de datado o período de 23000 a 2000 a.C. para a utilização das técnicas da MTC, há evidências fidedignas da utilização desses métodos somente a partir de 1523 a.C. (dinastia Shang).

\section{A chamada Medicina Clássica Chinesa} teve sua ascensão durante o período de 206 a. C., na dinastia HÀN até 220 d. C., sintetizando os pareceres de cosmologia, sociologia e medicina, declinando durante a dinastia QING (19441912) devido a influência cultural ocidental. Porém, após a revolução comunista de 1949, a equipe da estruturação da República Popular da China, na tentativa de resgatar a cultura tradicional da região cria a conhecida MTC. ${ }^{32}$

As técnicas enquadradas na MTC, dentre elas as técnicas de massagem (Tui-Na), exercícios respiratórios (Qi-Chong), orientações nutricionais (Shu-Shieh), acupuntura, fitoterapia chinesa (medicamento de origem animal, vegetal e mineral), ventosa, moxabustão, entre outras, possuem na sua essência estudar o organismo de forma geral buscando reestabelecer a saúde e fluidez energética. Ela baseia-se em observar a natureza e compreender sua harmonia, sendo assim, refere-se ao universo e ao homem como um todo, no qual ambos estão expostos aos mesmos impactos, podendo estabelecer uma semelhança entre a natureza e a fisiologia humana. ${ }^{36^{\prime}} 37$

Algumas técnicas terapêuticas da MTC podem ser explicadas por Mazer ${ }^{8}$, que se refere à acupuntura como uma técnica de introdução de agulhas em pontos dos meridianos do corpo, podendo ser dividida principalmente em sistêmica (corpo inteiro) e auricular (utilização de esferas no pavilhão da orelha). Já o moxabustão embasa-se no aquecimento desses pontos para ativar a fluidez da energia vital (Qi), a técnica de ventosa realiza através de copos que 
provocam uma pressão negativa com finalidade de restaurar o fluxo sanguíneo, e a fitoterapia que faz uso de plantas para encontrar o equilíbrio de Qi do organismo.

Segundo Maciocia $^{38}$, a MTC trata apenas a energia em desequilíbrio e não a doença. Para tanto, se faz necessário entender os conceitos ou teorias que cercam essas técnicas milenares, que abrangem três princípios básicos: a teoria do Yin e do Yang, dos cinco movimentos e do Zang Fu (Órgãos e vísceras). Essas teorias foram elaboradas pelos chineses devido ao costume de reparar os ciclos da natureza e suas alterações, sendo aplicadas na área médica para explicar a fisiologia e patologias do organismo. ${ }^{39} 40$

\section{TEORIAS DA MEDICINA TRADICIONAL CHINESA}

Brasil $^{30}$ retrata a MTC como um conjunto médico integral, onde se utiliza dos fenômenos da natureza e da interação harmônica para uma total integridade das partes, apresentando como parâmetro a teoria Yin e Yang que consiste em duas forças opostas, porém complementares, a teoria dos cinco movimentos que confere ao corpo uma das cinco energias (madeira, fogo, água, terra e metal), e o conceito Zang $\mathrm{Fu}$ (órgãos e vísceras) que trata da fisiologia energética dos órgãos e possibilita o entendimento da fisiopatologia das doenças e seu devido tratamento. ${ }^{30^{\prime}} 39^{\prime} 41$

\section{TEORIA YIN E YANG}

Conceitos da China antiga atribuem que a essência do ser humano era a mesma do universo, dividindo os fenômenos da natureza em dois polos distintos, porém que se completam os chamados Yin (negativo) e Yang (positivo). ${ }^{42}$ Para Maciocia ${ }^{38}$ a teoria de Yin- Yang foi elaborada a partir do estudo da mudança cíclica do dia e da noite, onde o dia (Yang) concede espaço à noite (Yin) e vice- versa, percebendo-se então a interferência contínua de uma energia sobre a outra. Para o autor, essa alternância cíclica, resulta em uma variação de forma, podendo ser exemplificada pela transformação da água em vapor pelo calor do dia, e a condensação do vapor pelo frio do cair da noite, tornando-o água novamente. Apoiando na teoria de dois estágios de transformação, pode-se dizer que Yang refere-se às coisas imateriais e energia pura, e o Yin retrata algo material.

$$
\text { As forças Yin e Yang estão }
$$


concomitantemente em um processo de oposição e interdependência. O processo de oposição pode ser explicado pela restrição e interação mútua e contínua, onde essa interação constante promove as mudanças e evolução dos objetos. Um exemplo aplicada a essa oposição seria a alteração das quatro estações do ano, onde o aumento do Yang Qi reduz o frescor do outono e frio do inverno, gerando a primavera morna e o verão quente. Seguindo esse padrão, as temperaturas diminuídas no outono e no inverno ocorrem devido ao aumento da força Yin que inibe o calor da primavera e verão. ${ }^{40}$

Apesar de opostas, as energias YinYang necessitam uma da outra para existir, compondo uma energia única, "Yin está instalado no interior como se fosse material básico do Yang, enquanto que o Yang permanece no exterior como uma manifestação da função Yin". 40

\section{Maciocia $^{38}$ e Mazer $^{8}$ citam que essa} oposição não é algo completo, e sim proporcional, bem como nem tudo é extremamente Yin ou Yang, mas tudo apresenta uma porção da sua oposição, expressando um equilíbrio dinâmico entre essas energias.

De acordo com Enomóto $^{43}$ a relação harmônica dessas duas energias é descrita com o termo Tao (Figura 1), onde o Yin e Yang se alternam em equilíbrio, ou seja, está em um constante consumo e aumento mútuo das energias. Nas atividades funcionais do organismo essa teoria de equilíbrio pode ser descrita pela presença de nutrientes para o funcionamento dos órgãos Zang-Fu, e esse funcionamento levará ao estímulo para a produção de mais Qi de defesa e nutricional. Em condições anormais desse consumo e aumento das energias, ocorrerá a desarmonia de Yin e Yang, provocando patologias (WEN, 1992). ${ }^{42}$

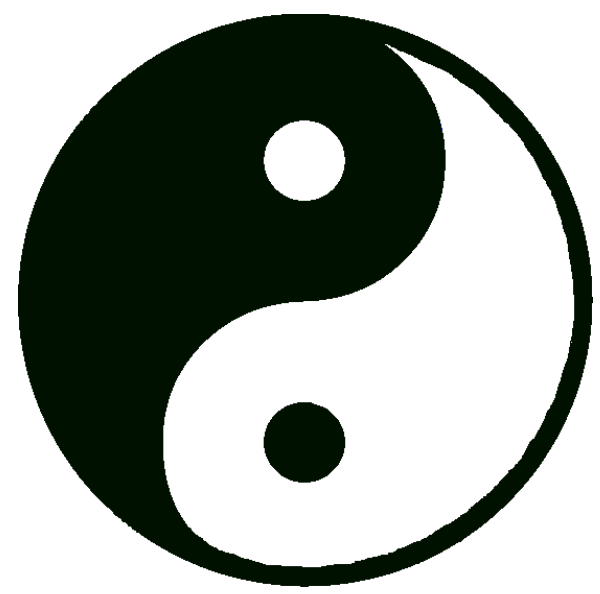

Figura 1. Tao. ${ }^{44}$

\section{TEORIA CINCO ELEMENTOS}

A segunda base para os princípios da MTC segundo Campiglia ${ }^{45}$ é a teoria dos cinco elementos, que se fundamenta na formação do universo através do movimento energético e modificação dos cinco conceitos: madeira, fogo, 
terra, metal e água, onde essas manifestações naturais sofrem ciclos de geração e dominação uns sobre os outros. ${ }^{8} 39$ O ciclo de geração (Figura 2) é explicado pela formação de um elemento a partir do outro que o antecede. Dessa forma o Fogo origina a Terra, que origina o Metal, que resulta na Água, que produz a Madeira, que volta ao Fogo. ${ }^{46}$

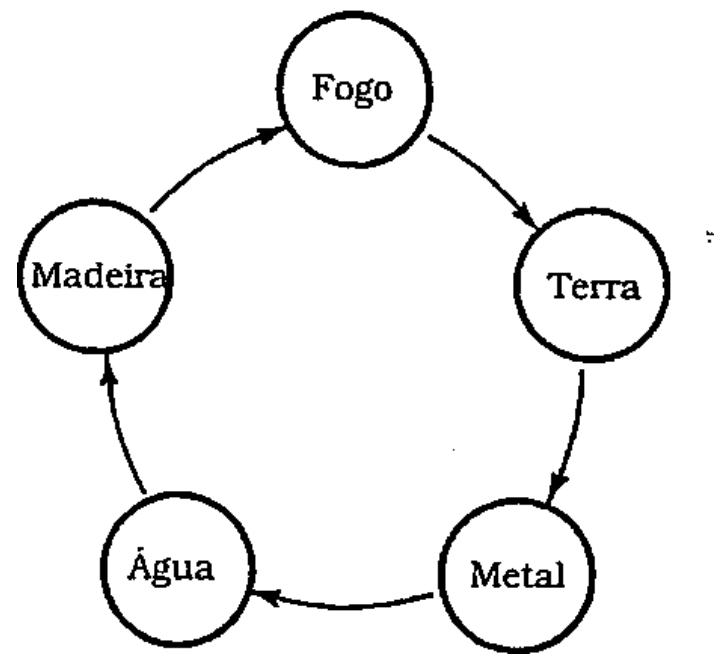

Figura 2. Ciclo de geração. ${ }^{38}$

Neste ciclo, a Madeira, por sua combustão, é capaz de gerar o Fogo, assim como promover sua intensidade. Após a combustão da Madeira, restam as cinzas, que são incorporadas à Terra. Ao longo dos anos, a Terra, sob o efeito de grandes pressões, produz os Metais. E dos metais e rochas brotam as fontes de Água. Por outro lado, a Água dá vida aos vegetais e, gerando a Madeira, fecha o ciclo da natureza. ${ }^{42}$
Já na chamada sequência de controle (Figura 3), cada componente dos fenômenos da natureza controla e é controlado para que haja uma estabilidade entre esses componentes. Neste ciclo, a Madeira controla a Terra, pois faz uso de seus nutrientes para o crescimento das árvores, além de modificar a estrutura do solo com suas raízes. A Terra dificulta a vertente de água, uma vez que a absorve e impede que se espalhe, a Água inibe o Fogo, fato esse bem compreensível, pois Água apaga o Fogo, e o elemento Fogo atrapalha o desenvolvimento do Metal, pois o derrete e o Metal, por fim, corta a Madeira, além de prejudicar o crescimento de raízes de árvores quando encontradas no solo, finalizando dessa forma mais um ciclo natural..$^{38^{\circ} 42}$

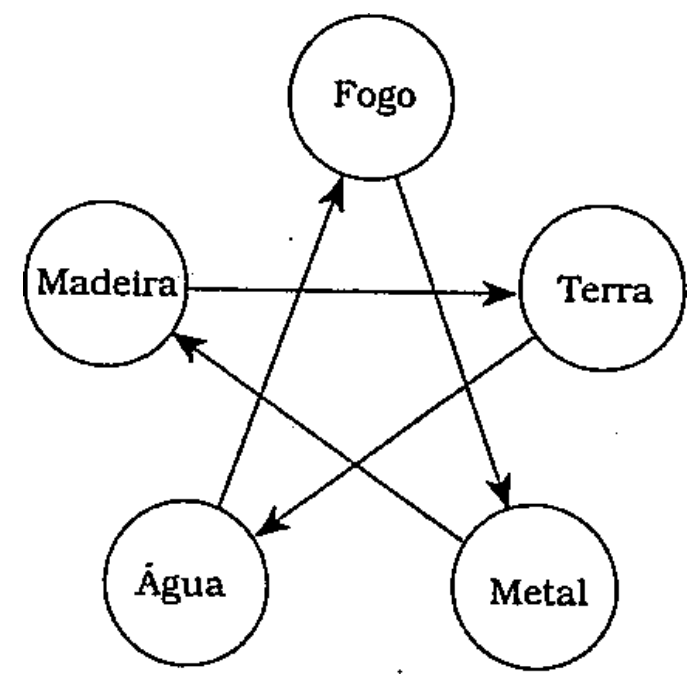

Figura 3. Sequência de Controle. ${ }^{38}$

$$
\text { Para Campiglia }{ }^{45} \text { e } \text { Enomóto }^{43} \text { a }
$$

Sequência de Dominância/Controle, quando se 
torna patológica, gera o ciclo de agressão (Figura 4), onde o elemento que deveria ser dominado, na verdade comanda o dominador do ciclo natural. Como exemplo, a Água é o comandante, no ciclo de controle, do Fogo, porém se Água apresentar-se reduzida, o Fogo inibirá a função da Água.

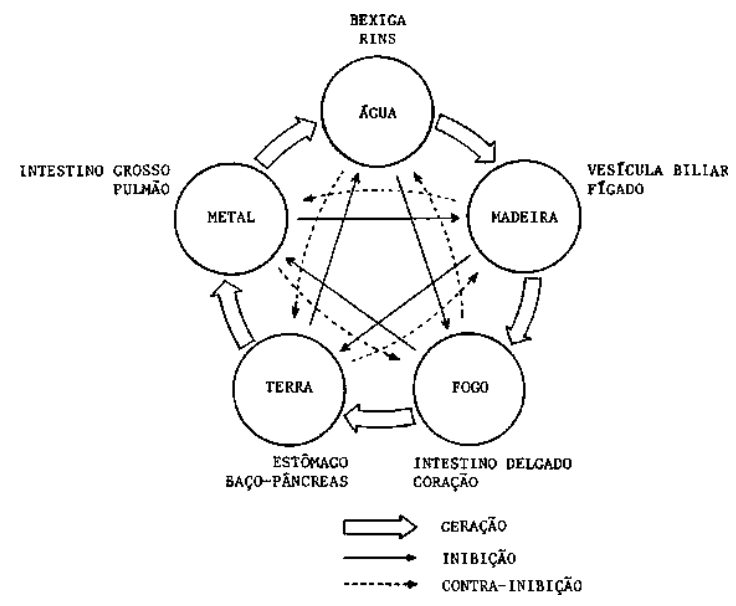

Figura 4. Três ciclos e os Cinco Elementos. ${ }^{42}$

Ainda para Campiglia ${ }^{45}$ a teoria dos cinco elementos está relacionada aos mais diversos aspectos, e não só apenas aos fenômenos da natureza, dentre eles estão os sabores, sons, cores, fisiologia do organismo e mente, e estão representados na Figura 5

\begin{tabular}{|c|c|c|c|c|c|}
\hline & Madeira & Fogo & Terra & Metal & Água \\
\hline Estaçôes & Primavera & Verão & Entre todas & Outono & Inverno \\
\hline Direções & Leste & Sul & Centro & Oeste & Norte \\
\hline Cores & Verde & Vermelho & Amarelo & Branco & Preto \\
\hline Sabores & Azedo & Amargo & Doce & Picante & Salgado \\
\hline Climas & Vento & Calor & Umidade & Secura & Frio \\
\hline Épocas da vida & Nascimento & \begin{tabular}{|l|} 
Crescimento \\
\end{tabular} & Transformação & Colheita & Estoque \\
\hline Sistemas Yin & $\begin{array}{l}\text { Fígado } \\
(\text { Gan })\end{array}$ & $\begin{array}{c}\text { Coração } \\
(\text { Xin })\end{array}$ & $\operatorname{Baço~}\left(P_{i}\right)$ & $\begin{array}{l}\text { Pulmão } \\
(F e t)\end{array}$ & $\operatorname{Rim}($ Shen $)$ \\
\hline Sistemas Yang & $\begin{array}{c}\text { Vês. Biliar } \\
\text { (Dan) }\end{array}$ & $\begin{array}{l}\text { Int. Delgado } \\
\text { (Xiaochang) }\end{array}$ & $\begin{array}{c}\text { Estômago } \\
(W e r)\end{array}$ & $\begin{array}{l}\text { Int. Grosso } \\
\text { (Dachang) }\end{array}$ & $\begin{array}{c}\text { Bexiga } \\
\text { (Panaavang) }\end{array}$ \\
\hline Órgãos dos Sentidos & Olhos & Língua & Boca & Nariz & Ouvido \\
\hline Tecidos & Tendốs & Vasos & Músculos & Pele, pếlos & Ossos \\
\hline Emoçôes & Fúria & Alegria & Preocupação & Tristeza & Medo \\
\hline
\end{tabular}

Figura 5. Correspondências dos Cinco Elementos. ${ }^{8}$

\section{TEORIA ZANG FU}

A teoria dos cinco elementos na MTC, segundo $\mathrm{Mazer}^{8}$, fundamenta os aspectos fisiológicos do corpo humano, auxiliando no diagnóstico e tratamento por essas técnicas. Enomóto $^{43}$ relata que para cada um desses elementos, existe um órgão (Zang) e uma víscera $(\mathrm{Fu})$, correspondentes as energias Yin e Yang. Esses órgãos e vísceras, na verdade, estão relacionados a compostos energéticos do organismo, e que interferem diretamente ou indiretamente no campo físico, mental e emocional do ser humano.

Auteroche, Navailh ${ }^{47}$ declara como os órgãos Zang o Coração, o Fígado, o Baço, o Rim e o Pulmão, com características Yin, apresentam funções de fornecer, estocar e converter as energias vitais. Já as vísceras $(\mathrm{Fu})$, o Intestino Delgado, Intestino Grosso, Triplo Aquecedor, a Vesícula Biliar e a Bexiga, com peculiaridades Yang, executam a eliminação de resíduos.

O órgão Fígado ou Gan, correspondente ao elemento Madeira, é responsável por armazenar o sangue (Xue) e simplificar a passagem de Qi, associando-se então a funcionalidade dos olhos e tendões, pois na ótica 
da fisiologia humana, o sangue tem funções de umidificar os olhos e promover a elasticidade aos tendões por meio de sua hidratação. ${ }^{38 ’} 48$ Este órgão administra a energia Hun ou alma etérea, e através dela é possível a conceber plano, criar objetivos e sonhar, sendo que a ação desses planos é realizada pela Vesícula Biliar, víscera complementar ao Fígado. ${ }^{49}$

O Coração ou Xin, órgão representante do Fogo, é encarregado de transportar o sangue (Xue) pelos vasos e auxiliar na função do BaçoPâncreas (Pi). Neste órgão, se faz presente a energia Shen ou também conhecida como energia vital, energia essa que faz do ser humano mais consciente e comunicativo. O Coração possui como víscera o Intestino Delgado (Xiao Chang), capaz de obter e desenvolver as coisas, assim como ocorre com os alimentos, separa os nutrientes e distribui seus resíduos a outras vísceras. ${ }^{38^{\prime} 45^{\prime} 47^{\prime} 49}$

Já o elemento Terra é representado pelo órgão Baço-Pâncreas (Pi), com funções realizadas sobre os alimentos, e a manutenção da fluidez do Xue nos vasos, este órgão é associado com tecidos e membros do corpo humano. ${ }^{48}$ Sua energia Yi ou inteligência representa a memória, pensamentos e intenções. O estômago (víscera do elemento Baço-Pâncreas) realiza a digestão dos alimentos, e juntamente com o órgão complementar Yin, separam a essência dos alimentos, para poder nutrir o organismo..$^{45^{\prime} 47}$

$$
\text { O Pulmão (Fei) comanda o Qi da }
$$
respiração e a propagação e descida do Qi do organismo. O espírito deste órgão é conhecido como Po ou alma corpórea, e suas características são os reflexos, os chamados sexto sentidos ou instintos, causador das reações de pânico ou fuga. A víscera Fu deste elemento é o Intestino Grosso (Da Chang), receptor e eliminador dos resíduos do Intestino Delgado. ${ }^{47} 48$

E por fim, o órgão representante da Água, os Rins (Shen). Eles estocam a essência vital (Jing), junção da essência recebida pelos pais e a essência adquirida pela absorção dos nutrientes dos alimentos, favorecem todo o processo de desenvolvimento desde o crescimento à reprodução, oferecem a excreção urinária e influenciam nas mais diversas estruturas e funções do organismo. ${ }^{48}$ A energia Zhi deste elemento promove o querer, a invenção, a habituação as diversas situações do dia-a-dia. Possui a Bexiga como víscera que conserva e expulsa os líquidos corporais. ${ }^{47 ’} 49$

Dessa forma, aliando a teoria Zang Fu com a teoria dos cinco elementos e suas sequências entende-se a conexão entre os órgãos 
e a influência que cada um exerce sobre o outro, podendo relatar que a energia essencial (Jing) dos Rins, nutre o órgão Fígado que por sua vez armazena o Xue auxiliando na funcionalidade do Coração, que intensifica a função do Baço, modificando a essência dos alimentos para encher os Pulmões, para promover a purificação e fluidez, ajudando na circulação da Água dos Rins. $^{8}$

\section{AURICULOTERAPIA}

A auriculoterapia é uma técnica aplicada em tratamentos baseados na MTC, no qual dispõe de pontos pré-estabelecidos localizados no microssistema auricular. Esses pontos já eram citados em um dos primeiros livros da MTC, conhecido como Di Nei Jing. ${ }^{43}$

Dito isso, define-se a auriculoterapia com uma terapia que explora os sinais da orelha como um método diagnóstico e de tratamento das enfermidades através da punção dos pontos por meio de agulhas, sementes de mostarda, esferas de cristais, prata e ouro para estabelecer o reequilíbrio fisiológico. ${ }^{8}$

Apesar de relatos históricos demonstrarem a sua existência há milhares de anos, foi com os estudos de Paul Nogier, em aproximadamente 1950, que esse método ficou mais conhecido e teve sua expansão até os dias de hoje. As pesquisas de Nogier tiveram como impulso a observação de pacientes que apresentavam cicatrizes na orelha para o tratamento da ciatalgia, fazendo com que o médico buscasse a relação de novos pontos na orelha e que estivessem interligados a partes distantes do corpo, comparando a orelha com a figura de um feto na posição invertida (Figura 6). ${ }^{50}$

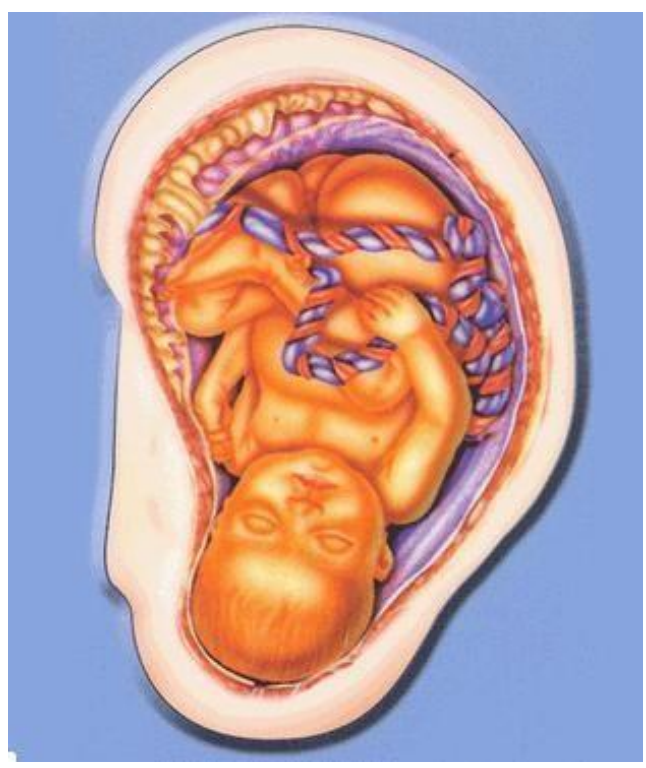

Figura 6. Comparação da orelha com feto invertido. ${ }^{51}$

A partir daí, estudos feitos por pesquisadores na China, beneficiaram-se das ideologias de Nogier e dos princípios das práticas chinesas para a criação de um novo mapa auricular, ramificando a auriculoterapia em duas escolas: a francesa e a chinesa. ${ }^{52}$ 
Segundo $\operatorname{Garcia}^{53}$ a localização desses pontos é prontamente identificada devido às divisões da orelha ocasionada pelas suas depressões. Essas regiões são conhecidas como: hélix, anti-hélix, trago, anti-trago, lóbulo, raiz do hélix, tubérculo auricular, fossa triangular, entre outros, como pode ser observado na figura 7.

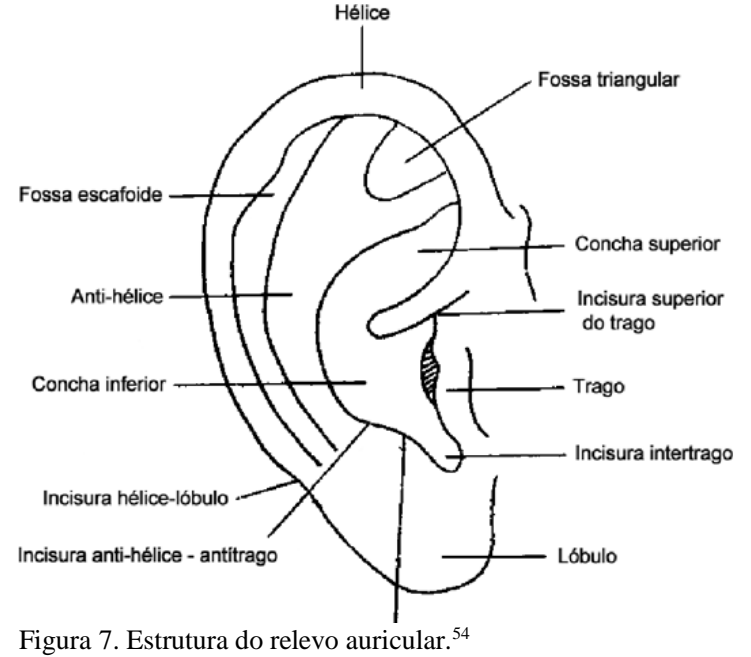

Como os pontos do mapa auricular expressam áreas específicas do corpo humano e sua fisiologia, torna-se possível visualizar se há alguma desordem ou patologia por meio de alterações morfológica, de coloração, edemas, vascularização visível, dor, descamação, e presença de nódulos. ${ }^{53}$

Partindo do princípio que a orelha apresenta respostas reflexivas de distúrbios energéticos nos órgãos internos, a primeira estratégia para a escolha dos pontos utilizados para os pacientes é a inspeção do pavilhão auricular na busca de alterações visíveis e descritas como um indicativo de patologia, já relatadas acima. ${ }^{55}$

Porém, Neves ${ }^{50}$ ressalta que a orelha acompanha o envelhecimento da pessoa, registrando as marcas da vida ao longo do tempo, portanto deve- se associar essas alterações juntamente com a identificação de pontos sensíveis à dor no exame de palpação e/ou vestígios do apalpador no pavilhão auricular, sempre seguindo uma ordem para o diagnóstico: anamnese, a inspeção e por último a palpação.

Analisando a anatomia da orelha é possível verificar que ela possui um número elevado de nervos e vasos sanguíneos, sendo que esses nervos tem origem craniana e espinhal, tornando-se responsáveis pelas reações provenientes da auriculoterapia, levando os estímulos às áreas do córtex cerebral e agindo sobre o sistema nervoso e consequentemente a todo o corpo. ${ }^{43^{\prime}} 50$

Para Landgren $^{56}$ a auriculoterapia atua sobre a liberação do cortisol e que torna um fator limitante sobre os níveis de estresse, agindo como um anti-inflamatório. Porém essa atuação só ocorre quando o estímulo provocado no microssistema ocorrer de forma mais suave, caso 
contrário, não haverá uma diminuição nos níveis de estresse.

\section{Discussão}

Após a leitura integral e avaliação dos 10 estudos selecionados, pode-se extrair as informações mais relevantes quanto ao objetivo do trabalho, que estão organizadas no quadro 1 (Anexo 1).

A respeito do objetivo dos estudos publicados, $100 \%$ apresentaram como foco principal mostrar a eficácia do tratamento para o estresse com intervenção da auriculoterapia, além de identificar os níveis de estresse na população de estudo. Destes, apenas $10 \%$ apresentou a diminuição do estresse a partir da auriculoterapia como único objetivo do trabalho, $30 \%$, compararam os pontos protocolares com o tratamento individualizado, $10 \%$ mostraram a diferença da aplicação do método por terapeutas mais experientes e menos experientes, $20 \%$ verificaram a auriculoterapia por meio de diferentes materiais, sendo eles agulhas versus sementes, 20\% relataram a diferença de aplicação entre pontos reais para o tratamento do estresse e pontos placebo, e por fim $10 \%$ demonstraram se o método é eficaz em desenvolver uma relação de empatia entre os cuidadores e pacientes, à medida que diminui o estresse destes profissionais.

Identificou- se como os principais pontos auriculares Shenmen, Rim e Tronco Cerebral (30\%), apresentando uma variação entre os estudos. Alguns utilizaram como protocolo os três pontos citados acima, com um acréscimo do Yang do Fígado 1 e 2 (30\%) (figura 8), 20\% fez uso apenas dos pontos Shenmen e Tronco Cerebral, $10 \%$ aplicou o método nos pontos Shenmen, pulmão, fígado e simpático e $10 \%$ não relataram os pontos nos quais foram aplicados a técnica.

$$
\text { Segundo Santos e Endo }{ }^{57} \text { os pontos }
$$
utilizados para o tratamento de estresse devem ter efeitos calmantes e sedativos como ocorre com os pontos Shenmen, Tronco Cerebral e Rim, o que explica a utilização desses pontos até mesmo para tratamentos individualizados nos estudos comparativos de protocolo (pontos estabelecidos sem a avaliação do paciente) e sem protocolo, onde foi realizada a anamnese e avaliação auricular para a definição dos pontos a partir da necessidade de cada paciente.

Maciocia $^{58}$ descreve a importância do ponto Rim para o tratamento do estresse, tendo em vista que rim funciona como abrigo do Qi, 
estimulando a vontade à vida, além de estar relacionado com sensações de medo e ansiedade.

O ponto Shenmen é caracterizado como sendo um ponto do sistema nervoso localizado na fossa triangular (metade superior do ápice) e é indicado para ações contra dor, sedação, problemas respiratórios, anti-inflamatório, inquietações mentais e insônia. O Rim está localizado na concha cimba, sob o começo da cruz inferior do hélix, sendo um ponto comumente usado para privação de vitalidade, distúrbios das vias urinárias, dores lombares, problemas de joelhos, coluna, audição, sentimentos de medo, cansaço, equilíbrio dos fluidos corporais, entre outros. Já o Tronco Cerebral localiza-se na fossa superior do antitrago, com funcionalidade de estimulação de mente e equilíbrio de sentimentos de pânico. O ponto Yang do fígado 1 e 2 , estão presentes na borda da hélice, com uso para controle de hipertensão e dores de cabeça. ${ }^{42^{\prime}}{ }^{50}{ }^{\prime} 53$

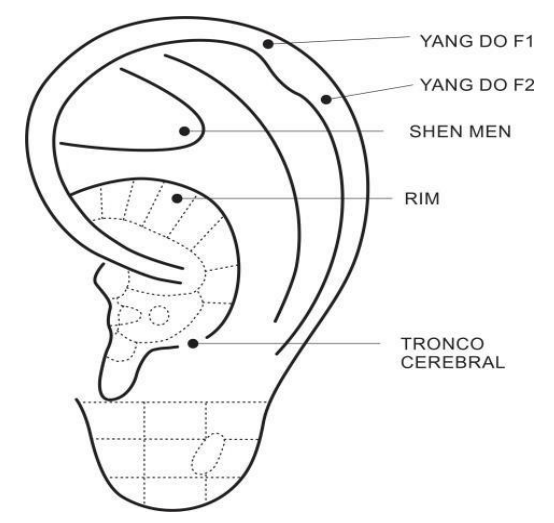

Figura 8. Localização dos pontos utilizados nas pesquisas. ${ }^{35}$
Todos os artigos tiveram como população de estudos indivíduos que trabalham ou irão trabalhar na área da saúde, sendo que $80 \%$ dos trabalhos selecionados foram executados em equipes de enfermagem (sendo que 1 artigo foi realizado em um grupo de estudantes de enfermagem), $10 \%$ em acadêmicos dos três últimos semestres de graduação de fisioterapia, e $10 \%$ em cuidadores da saúde, incluindo médicos, assistentes de atendimento, terapeutas, fonoaudiólogos, entre outros.

O fato da maioria dos estudos serem realizados em equipes de enfermagem pode ser explicado devido sua classificação, como sendo a quarta profissão mais estressante do âmbito público, devido as condições de trabalho em que essa população se encontra, com falta de reconhecimento, salários reduzidos, situações de empatia com pacientes que estão entre a vida e a morte, exigindo em aspectos físicos e emocionais destes profissionais, além de longas jornadas de trabalho e contato com materiais de $\operatorname{risco.}^{59^{\prime}} 60$

Duarte et al. ${ }^{24}$ traz também como fatores estressantes aos enfermeiros: locais artificiais, desde a iluminação ao ar condicionado, com ruídos o tempo todo, e a vivência em situações 
de dificuldades, dor e tristeza, além de estar em um ambiente que depende de suas habilidades e decisões para salvar vidas.

Seguindo essa linha, Rodrigues ${ }^{61}$ relata que além desses aspectos já citados a falta de recursos para que se possam pôr em prática as habilidades dos enfermeiros para com a vida, são responsáveis pelo aumento do estresse.

Em relação à população estudada, o quadro apresenta o total de pacientes que concluíram a pesquisa, por tanto alguns estudos não trazem a diferença de sexo entre os participantes (30\%), pois essa diferenciação não foi realizada, ou foi apenas abordada em uma população inicial da pesquisa. Para os estudos que trouxeram a porcentagem do sexo dos participantes iniciais, estes apresentaram um resultado semelhante aos trabalhos sintetizados neste estudo, nos quais há uma maioria de pessoas do sexo feminino (em $70 \%$ dos trabalhos).

Os níveis elevados de estresse em mulheres se dão devido à origem da profissão, que se apresenta em maioria para pessoas do sexo feminino pois, remete ao ato de cuidar, e ao possuírem dupla jornada de trabalho, o trabalho propriamente dito e os afazeres domésticos. ${ }^{59^{\prime}} 61$
Quanto ao número de sessões do tratamento por meio da auriculoterapia, pode-se constatar que $40 \%$ dos estudos empregaram a intervenção durante 12 sessões, $30 \%$ por 8 sessões, $10 \%$ houve discrepância nas informações, uma vez que o artigo aborda em sua discussão como se houvesse sido realizadas 12 sessões, porém nos procedimentos alega ter realizado apenas 8 sessões, $10 \%$ promoveram o tratamento por 7 sessões e por fim, $10 \%$ por 5 sessões. Para Neves ${ }^{50}$ o tratamento pela auriculoterapia deve ser realizado por um período de dez sessões, após isso aconselha- se um intervalo de aproximadamente 20 dias para a retomada de uma nova sessão.

Um estudo realizado por Prado, Kurebayashi, Silva ${ }^{62}$ intitulado de Eficácia da auriculoterapia na redução de ansiedade em estudantes de enfermagem, com uma amostra de 71 pacientes e objetivo de se comparar grupos de auriculoterapia e placebo, demonstrou que houve um resultado positivo quando realizado a primeira avaliação após 8 sessões, descrevendo ser o número suficiente de intervenções para se avaliar a técnica de auriculoterapia. Para tanto como a avaliação só foi realizada após esse período não se pode descrever a partir de qual sessão o tratamento começou a surtir efeito. 
Para um dos estudos analisados, onde os níveis de estresse foram medidos em 4 momentos (antes do início das intervenções, após quatro sessões, após 8 sessões e após 15 dias do término das sessões) observou-se uma redução de estresse significativa a partir da terceira avaliação, ou seja, após 8 sessões. Todavia, como pode- se observar alguns estudos trouxeram resultados positivos para quantidades inferiores a 8 sessões de intervenção pela auriculoterapia.

No que se diz respeito aos resultados das pesquisas, nota-se que em 100\% dos estudos analisados, houve uma resposta positiva ao uso da auriculoterapia para a redução dos sinais e sintomas de estresse, porém cada um com sua particularidade e objetivo diferenciado.

As interpretações dos resultados desses estudos só se tornaram possíveis, pois os autores fizeram uso de alguns instrumentos de acompanhamento para nivelar o estresse dos participantes ao longo das pesquisas, onde se apresentou a preferência pela utilização da Lista de Sintomas de Estresse de Vasconcellos (Anexo 2), sendo utilizado em $80 \%$ dos estudos com ou sem outros métodos de avaliação, e $20 \%$ dos estudos utilizaram outros métodos de avaliação. ${ }^{35}$
O LSS compreende 59 sinais do estresse, em nível psicológico, fisiológico e social do estresse, em que a pessoa analisada deve responder a existência e frequência de cada sintoma que se manifesta. Para cada sinal a pontuação varia de 0 a 3, podendo somar uma pontuação final de 0 a 177 pontos, no qual quanto maior a pontuação, maior será o nível de estresse. $^{63}$

Apesar de demonstrar uma eficácia na utilização da auriculoterapia para a diminuição dos níveis de estresse, houve algumas divergências com relação aos estudos comparativos entre tratamentos protocolares e individualizados, utilização de agulhas versus sementes, e resposta positiva para grupos placebos quando comparados com uma aplicação real do método.

Para os materiais que apresentaram uma resolução mais positiva aos tratamentos individualizados, esses podem ser explicados por Doria ${ }^{64}$ que alega que na MTC não existe uma única causa para o estresse e que o diagnóstico carece ser de forma individualizada, conforme os sintomas descritos por cada paciente, nas quais leva- se em consideração a história clínica, sinais, estilo de vida, entre outros, apesar de algumas características estarem 
frequentemente relacionadas ao estresse e desequilíbrios dos elementos e aos Zang Fu.

A eficácia superior para o tratamento com agulhas semipermanentes do que sementes pode ser justificada pela falta de estimulação dos pontos pelo pacientes, ao qual foi solicitado pelos terapeutas, no momento da consulta, por no mínimo 3 vezes ao dia. ${ }^{13}$

Prado, Kurebayashi, Silva ${ }^{62}$ trazem uma possível explicação para os resultados positivos para os grupos placebos, apesar de não terem sido significativos. Tal explicação baseia-se na presença de um ponto aproximado do ponto Ouvido externo, o ponto Interferon, conhecido no mapa francês como anti-inflamatório, levantando a importância da padronização do local e recomendação dos pontos na literatura, para estudos que avaliam pontos sham e pontos reais.

\section{Conclusão}

Através do estudo elaborado, foi possível evidenciar que a população foco de pesquisas sobre o estresse são profissionais da área de saúde, uma vez que a maioria está exposta a agentes estressores diariamente, na qual precisam lidar com situações difíceis como encarar a morte, e saber que este pode ser sua responsabilidade, seja ela por falta de conhecimento na área de atuação ou por condições inadequadas no local de trabalho.

Com relação a técnica aplicada nos estudos identificou-se uma utilização elevada dos pontos Shenmen, Rim, Tronco Cerebral e Yang do Fígado 1 e 2, tanto em grupos no qual o tratamento foi realizado com pontos já estabelecidos, quanto para o grupos que passaram por uma avaliação com o terapeuta para a escolha dos pontos de maior necessidade. Porém, recomenda-se o tratamento individualizado, onde seja feita a anamnese, a inspeção e pressão auricular, pois pode-se avaliar de forma mais eficaz os desequilíbrios energéticos de cada paciente, visando que cada pessoa é um ser único apresentando necessidades diferentes uma das outras.

A auriculoterapia apresentou um resultado positivo como método alternativo para a diminuição dos níveis de estresse. Para tanto, recomenda-se a técnica como forma de combate ao estresse, pois além de apresentar eficácia é um método de baixo custo e de fácil acesso nos dias de hoje. 


\section{Referências}

1- $\quad$ Favassa CTA, Armiliato N, Kalinine I. Aspectos Fisiológicos e Psicológicos do Estresse. Revista de Psicologia da UnC. 2005;2 (2): 84-92.

2- Lipp MEN. Stress no Trabalho: Implicações para a pessoa e para a empresa. Rio de Janeiro: Zit Editora; 2005.

3- Selye H. Stress: A tensão da vida. São Paulo: Ibrasa Editora; 1965.

4- $\quad$ Lipp MEN, Malagris LNO. Stress Emocional e seu Tratamento. In B. Range (Org). Terapias Cognitivo- Comportamentais: um diálogo com a psiquiatra. São Paulo: Artes Medicas; 2001.

5- Guido LA, Linch GFC, Pitthan LO, Umann J. Estresse, coping e estado de saúde entre enfermeiros hospitalares. Revista da Escola de Enfermagem da USP. 2011;45(6): 1434-1439.

6- $\quad$ Kurebayashi LFS, Gnatta JR, Borges TP, Silva MJP. Eficácia da auriculoterapia para estresse segundo experiência do terapeuta: ensaio clínico. Acta Paul Enferm, 2012;25(5):694-700.

7- Brasil. Ministério da Saúde. Política Nacional de Práticas Integrativas e Complementares no SUS: PNPIC: atitude de ampliação de acesso. Brasília, 2006b.

8- Mazer E. Apostila de auriculoterapia chinesa. Curso de formação em auriculoterapia, 2011. [cited 2016 Set 18] Available from: https://pt.scribd.com/document/57841328/apost ila-de-auriculoacupuntura-chinesa

9- Sarturi F. Nível de estresse do enfermeiro hospitalar frente as suas competências [dissertação] [internet].Santa Maria: Universidade Federal de Santa Maria; 2009. [cited 2016 Set 06]. Available from: http://docplayer.com.br/11019484-Nivel-destress-do-enfermeiro-hospitalar-frente-a-suascompetencias.html

10- Silva JJ. Stress o impulso da vida. São Paulo: $\quad$ Yendis; 2005.
11- Rossi AM. Autocontrole: uma nova maneira de gerenciar o estresse. Rio de Janeiro: Best Seller; 2006.

12- Ferreira ABH. Novo Dicionário da Língua Portuguesa. 3ed. Curitiba: Positivo; 2004.

13- Kurebayashi LFS, Gnatta JR, Borges TP, Belisse G, Coca S, Minami A, et al. Aplicabilidade da auriculoterapia com agulhas ou sementes para diminuição de estresse em profissionais de enfermagem. Rev Esc Enferm USP. 2012;46(1):89-95.

14- Luz C. Análise dos Mecanismos Psiconeuroimunoendócrinos que Regulam a Imunidade Celular em Idosos Saudáveis [tese]. Porto Alegre: Pontífica Universidade Católica do Rio Grande do Sul; 2006.

15- Morini CAT. Ativação bioenergética em meio líquido: Stress e qualidade de vida. Jundiaí: Ápice; 1997.

16- Trindade LL, Lautert L. Síndrome de Burnout entre os trabalhadores da Estratégia de Saúde da Família. Rev Esc Enferm USP. 2010;44(2):274-279.

17- Lipp MEN. Stress e suas implicações. Estudos de Psicologia. 1984;1(3-4):5-19.

18- Filho MGB, Ribeiro LCS, Miranda R, Teixeira MTA. Redução dos níveis de cortisol sanguíneo através da técnica de relaxamento progressivo em nadadores. Revista Brasileira de Medicina do Esporte. 2002;8(4):139-143.

19- Lipp MEN. Manual do inventário de sintomas de stress para adultos. São Paulo: Casa do Psicólogo; 2000.

20- Lazarus R, Folkman S. Stress, Appraisal and Coping. New York: Springer Publishing Company; 1984.

21- Limongi-frança AC. Psicologia do Trabalho: Psicossomática, Valores e Práticas Organizacionais. São Paulo: Saraiva; 2008.

22- Deliberato PCP. Fisioterapia preventiva: fundamentos e aplicações. São Paulo: Manole; 2002. 
23- Selye H. The Stress of Life. New York: McGraw-Hill;

1956.

24- Duarte AP, Nunes SMP, Oliveira AA. Prevalência da síndrome de burnout em enfermeiros atuantes em UTI. Psicologia.PT O Portal dos Psicólogos [Internet]. 2013 Jun [cited 2016 Out 13]; Availavle from: http://www.psicologia,pt/artigos/ver_artigos.ph p?codigo=A0696 ISSN 1646-697.

25- Meneghini F, Paz AA, Lautert L. Fatores Ocupacionais associados aos componentes as síndromeS de burnout em trabalhadores de enfermagem. Texto Contexto Enferm. 2011;20( 2):225-33.

26- Robbins S. Comportamento Organizacional. Rio de Janeiro: Editora LTC; 1999.

27- Silva DP. Trabalhador com estresse: Possibilidades de intervenção da terapia ocupacional [monografia]. Ceilândia: Universidade de Brasília; 2013.

28- Araújo APS, Zampar R, Pinto SM. Auriculoterapia no tratamento de indivíduos acometidos por distúrbios osteomoleculares relacionados ao trabalho (dort)/lesões por esforços repetitivos (ler). Arq. Ciênc. Saúde Unipar, Umuarama. 2006;10(1):35-42.

29- Caminha R, Wainer $\mathrm{R}$, Oliveira $\mathrm{M}$, Piccolato N. Psicoterapias cognitivocomportamentais teoria e prática. São Paulo: Casa do Psicólogo; 2007.

30- Brasil, Portaria n.971, de 03 de maio de 2006. Aprova a Política Nacional de Práticas Integrativas e Complementares (PNPIC) no Sistema Único de Saúde. Diário Oficial da União. $\quad 03 \quad$ mai 2006.

31- Luz MT. Cultura contemporânea e medicinas alternativas: Novos paradigmas em saúde no fim do século XX. Physics: Revista de Saúde Coletiva. 2005;15 Suple: 145-176.

32- Souza EAA, Luz MT. Bases Socioculturais das práticas terapêuticas alternativas. História, Ciências, Saúde Manguinhos. 2009;16(2): 393-405.

33- WHO - World Health Organization.
Tradicional Medicine Strategy: 2002-2005. Geneva: WHO, 2002.

34- Jayasuriya A. As bases científicas da Acupuntura. Rio de Janeiro: SOHAKU-IN Edições;

1995.

35- Kurebayashi LFS. Auriculoterapia Chinesa para redução do estresse e melhoria de qualidade de vida de equipe de enfermagem: ensaio clínico randomizado[tese]. São Paulo: Universidade de São Paulo; 2013.

36- Altman S. Acupuncture as an emergency treatment. California veterinarian. 1979;15(1):68.

37- Branco CA, Fonseca RB, Oliveira TD, Gomes VL, Fernandes Neto AJ. Acupuntura como tratamento complementar nas disfunções temporomandibulares: revisão da literatura. Revista de Odontologia da UNESP. 2013;34(1):11-6.

38- Maciocia G. Os fundamentos da Medicina Chinesa. São Paulo: Roca; 1996.

39- Yamamura Y. Acupuntura tradicional e a arte de inserir. São Paulo: Roca; 2001.

40- Junying G, Zhihong S. Medicina tradicional chinesa prática e farmacologia: teorias e princípios básicos. São Paulo: Roca; 1996.

41- Lipp MEN, Malagris LN. Stress ao longo da vida. São Paulo: Ícone; 2007.

42- Wen TS. Acupuntura clássica chinesa. São Paulo: Cultrix; 1992.

43- Enomóto J. Auriculoterapia: Método Enomóto. São Paulo: Ícone; 2015.

44- Nascimento CA. Acunpuntura como terapia complementar no tratamento dos sintomas do estresse [monografia]. São José dos Campos: Faculdade de Educação Ciência e Tecnologia;

2013.

45- Campiglia H. Psique e medicina tradicional chinesa. São Paulo: Roca; 2004.

46- Cordeiro A, Cordeiro RC. Acupuntura elementos básicos. São Paulo: Polis; 2001. 
47- $\quad$ Auteroche B, Navailh P. O diagnóstico na medicina chinesa. São Paulo: Andrei; 1992.

48- WHO - World Health Organization. Regional Office for the Western Pacific. WHO International Starndard Terminologies on Traditional Medicine in the Western Pacific Region. Geneva; 2007

49- Hicks A, Hicks J, Mole P. Five Constitucional Acupuncture. London: Churchill Livingstone;

2011.

50- Neves ML. Manual prático de auriculoterapia. Porto Alegre: Ed. Do Autor; 2016.

51- Hermes R. Feto invertido. Auriculoterapia: 2012. [acesso 31 Out. 2016] Disponível em: http://www.renatahermes.com.br/2012/08/auric uloterapia/

52- Lopes SS. Analgesia por Acupuntura. Curitiba: Ommipax; 2013.

53- Garcia E. Auriculoterapia. São Paulo: Roca;

1999.

54- Capdet PPA. Estrutura do relevo auricular. Guia para encontrar os pontos da acupuntura auricular chinesa. [acesso 02 Nov. 2019] Disponível em: http://www.acupuntura$\mathrm{mtc} . c o m / t e r a p i a s-m t c / p o n t o s-a c u p u n t u r a$ auricular.php

55- Abbate S. Chinese Auricular Acupuncture. New York: CRC PRESS; 2003.

56- Landgren K. Explanatory models for acupuncture. Ear Acupuncture: a practical guide. New York: $\quad$ Elsevier; 2008.

57- Santos GO, Endo EE. Auriculoterapia e Estresse: Avaliação da efetividade e tempo de duração de uma sessão. São Paulo: Instituto de Terapia Integrada e Oriental; 2009. [acesso 02 Out. 2016] Disponível em:

http://www.itiomassagem.com.br/artigos/mono grafias/item/77- auriculoterapia-e-estresseavaliacao-da-efetividade-e-tempo-de-duracaode-uma-sessao-sao-paulo-sp-2009

58- Maciocia G. Os fundamentos da medicina chinesa. São Paulo: Roca; 2007.

59- $\quad$ Ferreira LRC, Martino MMF. Stress no cotidiano da equipe de enfermagem e sua relação com o cronótipo. Estudos de Psicologia. 2009; 26(1):

65-72.

60- Silva DP, Silva MNRO. O trabalhador com estresse e intervenções para o cuidado em saúde. Trabalho, Educação e Saúde. 2015; 13 (Suppl.1):

201-214.

61- Rodrigues VMCP, Ferreira ASS. Fatores geradores de estresse em enfermeiros de Unidades de Terapia Intensiva. Revista Latino Americana de Enfermagem. 2011;9(4): 10251032.

62- $\quad$ Prado JM, Kurebayashi LFS, Silva MJP. Eficácia da auriculoterapia na redução de ansiedade em estudantes de enfermagem. Rev Esc Enferm USP. 2012;46(5):1200-1206.

63- Ferreira EAG, Vasconcellos E, Marques AP. Assessment of pain andstress in fibromyalgia patients. Revista Brasileira de Reumatologia. 2002; $42(2)$.

64- Doria MCS. O uso da acupuntura na sintomatologia do stress [dissertação]. Campinas: Pontífica Universidade Católica; 2010.

Data de submissão: 20/11/2019

Data de aprovação: 25/02/2020 
Acta Elit Salutis- AES - (2020) v.2 (1)

ISSN online 2675-1208 - Artigo Original 